\title{
The use of zoledronic acid in Japanese men with stage $\mathrm{D} 2$ prostate cancer
}

\author{
KOGENTA NAKAMURA ${ }^{1}$, YOSHIAKI YAMADA ${ }^{1}$, CHARLES J. ROSSER ${ }^{3}$, MAKI ARAKAWA ${ }^{2}$, \\ KENJI ZENNMAI ${ }^{1}$, YOSHIHARU KATO ${ }^{1}$, MASAHITO WATANABE ${ }^{1}$, REMI KATSUDA ${ }^{1}$ \\ MOTOI TOBIUME ${ }^{1}$, KATSUYA NARUSE ${ }^{1}$, SHIGEYUKI AOKI ${ }^{1}$, TOMOHIRO TAKI ${ }^{1}$, \\ HIROKO SAITO $^{2}$, TAKAAKI HASEGAWA ${ }^{2}$ and NOBUAKI HONDA ${ }^{1}$ \\ ${ }^{1}$ Department of Urology, Aichi Medical University School of Medicine, Nagakute-cho, Aichi 480-1195; \\ ${ }^{2}$ Department of Hospital Pharmacy, Aichi Medical University Hospital, Nagakute, Aichi 480-1195 Japan; \\ ${ }^{3}$ Department of Urology, University of Florida, Gainesville, FL 32610, USA
}

Received January 20, 2009; Accepted June 30, 2009

DOI: 10.3892/ol_00000002

\begin{abstract}
Zoledronic acid (ZOL) is a new generation bisphosphonate with improved efficacy benefits over pamidronate in preclinical testing. In addition, $\mathrm{ZOL}$ is superior to pamidronate in the treatment of hypercalcemia of malignancy. ZOL is also the first bisphosphonate to demonstrate efficacy in patients with bone metastases from solid tumors other than breast cancer, such as prostate cancer. In this study, we investigated ZOL treatment in 17 Japanese men with advanced prostate cancer, treated at the Aichi Medical University Hospital between August 2006 and November 2007. The 17 patients had biopsy-confirmed prostate cancer and were found to harbor bone metastasis upon bone scintigraphy. ZOL was administered intravenously at a dose of $4 \mathrm{mg}$ over 15 min every 4 weeks. ZOL was well tolerated with mild renal dysfunction in 2 patients $(11.8 \%)$, while 1 patient $(5.8 \%)$ developed skin rash. No significant side effects were observed. Subjective improvement in bone pain was reported in 14 patients $(32.4 \%)$. ZOL, therefore, is a safe and effective drug that remains an important component of the urologist's armamentarium against advanced prostate cancer.
\end{abstract}

\section{Introduction}

Prostate cancer is one of the most common types of cancer in men worldwide $(1,2)$. Although stage migration of prostate cancer has been reported, when metastatic disease occurs it usually involves the bones $(3,4)$. In addition to bone metastases, bone demineralization from previous castration (medical or

Correspondence to: Dr Kogenta Nakamura, Department of Urology, Aichi Medical University School of Medicine, Nagakute-cho, Aichi 480-1195, Japan

E-mail:kogenaka@aichi-med-u.ac.jp

Key words: zoledronic acid, advanced prostate cancer, bone metastasis surgical) contributes to an increased risk of skeletal complications (e.g., fracture and pain) (5-8). Complications from bone metastases are a major cause of morbidity in patients with prostate cancer, causing intractable debilitating pain, spinal cord compression, pathologic fractures and abnormalities in serum calcium levels (9). Thus, patients with metastatic hormone-refractory prostate cancer (HRPC) are particularly prone to incapacitating progressive bone disease (10).

Zoledronic acid (ZOL) is a new generation nitrogen-containing bisphosphonate (BP) with improved efficacy benefits over pamidronate in preclinical testing. In addition, ZOL is superior to pamidronate in the treatment of hypercalcemia of malignancy (11). It is also the first BP to demonstrate efficacy in patients with bone metastases from solid tumors other than breast cancer, including prostate cancer, non-small cell lung cancer and a variety of other tumor types $(12,13)$. Although not an oral agent, ZOL can be safely administered intravenously (14) (Maxwell et al, Convenience of a 15-minute infusion of zoledronic acid. Presented at the 3rd European Oncology Nursing Symposium Spring Convention, Venice, Italy, April 12-14, 2002).

ZOL has been evaluated in phase I trials in patients with a variety of cancer types and bone metastases $(15,16)$. In a previous comparative phase III trial (17), a 4-mg infusion of ZOL was as effective as a $90-\mathrm{mg}$ infusion of pamidronate in reducing skeletal complications in patients with multiple myeloma or breast cancer. Previously, Saad et al reported that ZOL treatment for metastatic hormone-refractory prostate cancer significantly reduced the incidence of skeletal-related events (SRE) by 36\%, and delayed the first SRE by $>5$ months as compared to the placebo used (18). Moreover, BPs are well tolerated with only minimal side effects (e.g. influenza-like syndrome, arthralgia and gastrointestinal symptoms). We investigated ZOL treatment in 17 Japanese men with advanced prostate cancer, treated at the Aichi Medical University Hospital between August 2006 and November 2007. Specifically, we evaluated the effects of ZOL on SRE, bone pain, adverse events, serum calcium, creatinine and prostate-specific antigen (PSA) in subjects with advanced prostate cancer. 
Table I. Clinicopathological characteristics of the cohort.

\section{Patient no.} 17

Age (years)

TNM classification (UICC ${ }^{\text {a) }}$

T2aNOM1b

T2bN0M1b

T3aN1M1b

T3bN1M1b

T4N0M1b

T4N1M1b

Peformance status $\left(\mathrm{ECOG}^{\mathrm{b}}\right)$

PS 0

PS 1

PS 2

PS 3

\section{HRPC $^{c}$}

Non-HRPC

Previous SRE ${ }^{\mathrm{d}}$

Yes

No

Prior to $\mathrm{BP}^{\mathrm{e}}$

Yes

No

6 (incadronate disodium) 11

aUICC, International Union against Cancer; 'ECOG, Eastern Cooperative Oncology Groups; 'HRPC, hormone-refractory prostate cancer; ${ }^{\mathrm{d} S R E}$, skeletal-related events; ${ }^{\mathrm{B} P}$, bisphosphonate.

\section{Patients and methods}

Study population. Our cohort comprised of 17 non-consecutive patients with biopsy-confirmed stage D2 prostate cancer and bone metastasis; verified by bone scintigraphy. Table I lists the demographic and clinicopathological characteristics of the cohort. Of the 17 patients, 12 had HRPC, while 5 were sensitive to androgen manipulation. HRPC patients had received docetaxel-based chemotherapy or steroidal therapy. NonHRPC patients had received combined androgen blockade therapy. Patients had been prescribed morphine and nonsteroidal anti-inflammatory drugs (NSAIDS) for moderate to severe bone pain. Six patients were previously treated with incadronate disodium. Three patients had SRE (1 patient with a compression fracture and paralysis and 2 patients with pending fractures, which were treated with radiation therapy). No patients were found to have hypercalcemia of malignancy.

Methods. ZOL (Zometa ${ }^{\circledR}$; Novartis Pharma AG, Basel, Switzerland/Novartis Pharmaceuticals Corp., East Hanover, $\mathrm{NJ}$ ) was administered intravenously at a dose of $4 \mathrm{mg}$ over 15 min every 4 weeks (one cycle). Treatments ranged from 1 to 13 cycles (median of 6 cycles). Hydration was not performed. Clinical and hospital records were reviewed for several key fac-



Figure 1. PSA values were evaluated prior to (PreZOL), 1 month after (PostZOL 1M) and a few months after (PostZOL) ZOL injection.



Figure 2. Changes in serum creatinine levels prior to (PreZOL) and within a few days after (PostZOL) ZOL injection.

tors including laboratory results and SRE. Statistical analysis of the laboratory results was evaluated using the Wilcoxon and Friedman's tests. The patients were assessed for any adverse effects using the National Cancer Institute's Common Toxicity Criteria, version 2.0. Pain complaints were evaluated. The median follow-up was 11 months.

\section{Results}

Although serum PSA levels were not altered (Fig. 1), a significant reduction in subjective bone pain was evident. Subjects previously utilizing morphine and NSAIDS for moderate to severe bone pain, reported that bone pain decreased in (14 subjects, $82.4 \%$ ) with ZOL treatment. The other three subjects, however, reported a dose escalation of morphine to control bone pain. No new SRE was evident in the cohort.

ZOL therapy was well tolerated in our cohort. Mild renal dysfunction (i.e., serum creatinine 1.4-2.0 mg/dl) was detected in two patients $(11.8 \%)$. However, no statistically significant differences were noted in serum creatinine levels between pre- and post-ZOL injection ( $\mathrm{P}=0.06)$ (Fig. 2). Skin rash was developed in one patient $(5.8 \%)$. The two patients with renal dysfunction were on medication for hypertension. No sig- 


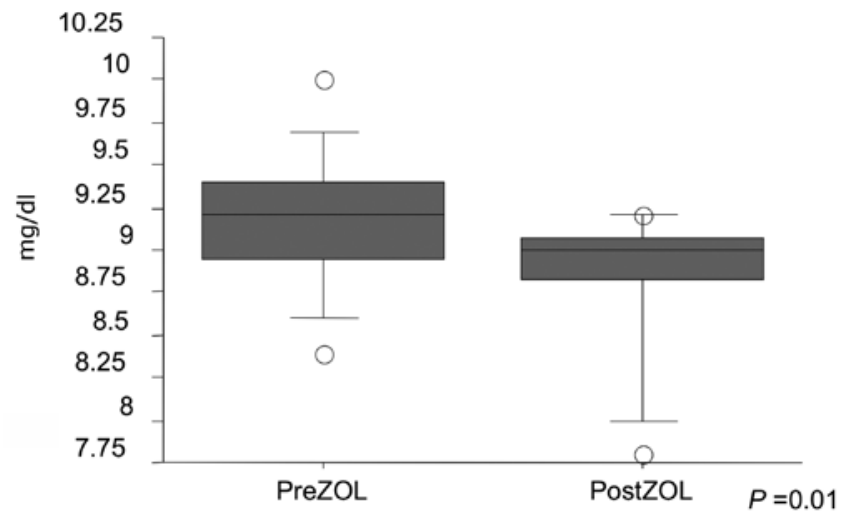

Figure 3. Changes in serum calcium levels prior to (PreZOL) and within a few days after ZOL injection (PostZOL).

nificant side effects were noted. As for abnormalities in serum chemistry, serum calcium decreased post-ZOL injection $(\mathrm{P}=0.01)$, but there was no evidence of severe hypocalcemia (Fig. 3).

\section{Discussion}

Bone metastasis in prostate cancer can lead to significant skeletal morbidity. In randomized controlled trials, BP therapy has been associated with a reduction in SRE. BPs are known to reduce excessive bone turnover while preserving bone structure and mineralization in patients with metastatic disease to the skeleton (15). We investigated the efficacy of ZOL in a cohort of Japanese men with stage D2 prostate cancer.

We found no SRE in the small cohort of Japanese men with stage D2 prostate cancer treated with ZOL. Previously, Saad et al reported that ZOL significantly reduced the incidence of SRE by $36 \%$ and delayed the first SRE by $>5$ months as compared to the placebo used (18). Furthermore, ZOL provided significant long-term reductions in bone pain as compared to the placebo used (19). Unlike ZOL, first generation BPs (etidronate, clodronate and pamidronate) were unable to demonstrate a statistically significant clinical benefit in patients with bone metastatic prostate cancer (15). Previous reports and the present study show that ZOL significantly lowers the incidence of skeletal complications and helps in alleviating bone pain. Thus, ZOL is currently the most commonly chosen BP treatment for patients with hormone-refractory bone metastatic prostate cancer. ZOL subjectively improved bone pain in 14 patients $(82.4 \%)$ who were previously treated with morphine and NSAIDS. In 5 cases, morphine and NSAID treatment decreased. However, in our cohort, 3 patients reported an increase in morphine use to alleviate bone pain. Notably, SRE reduction was greatest in patients without pain. Thus, it is advised that ZOL therapy be started before patients present symptoms (20).

Several issues related to BP therapy are worth mentioning. First, it is not uncommon for patients to experience an increase in creatinine levels while on therapy. Vogel and others noted a mild increase in serum creatinine in $7.7 \%$ of patients, previously treated with BP therapy, as well as $4.5 \%$ of BP-naïve patients (16). In our cohort (11.8\%), ZOL therapy resulted in mild and transient renal dysfunction (increase in serum creatinine from 1.4 to $2.0 \mathrm{mg} / \mathrm{dl}$ ). Second, BP therapy has been associated with severe hypocalcemia (21). We found a non-significant decrease in serum calcium levels noted within a few days of BP therapy. Severe hypocalcemia, however, was not noted. On the other hand, Gulley et al reported that following a single dose of zoledronic acid, one patient developed hypocalcemia which persisted for approximately 60 days (21). Third, osteonecrosis of the jaw has been linked to BP therapy (22). In our study, BP treatment was delayed in one subject to accommodate for dental evaluation and treatment. It is therefore critical that patients receiving BP be taught meticulous dental care to prevent osteonecrosis of the jaw. Fourth, ZOL can halt bone turnover associated with metastatic disease to the bones. Previously, Vordos and colleagues reported that ZOL therapy in combination with docetaxel was associated with a decrease in serum PSA by more than $50 \%$ at 2 months in more than $50 \%$ of the patients (23). Thus, a significant reduction in PSA may be associated with burden of disease within the skeleton. We noted no decrease in patient serum PSA levels during ZOL therapy. Significant clinical benefits of ZOL therapy were demonstrated in this retrospective analysis of 17 Japanese men with bone metastasis from prostate cancer. It is therefore critical that urologists understand the benefits and potential implications of $\mathrm{ZOL}$ in the treatment of stage $\mathrm{D} 2$ prostate cancer.

\section{References}

1. Parkin DM, Laara E and Muir CS: Estimates of the worldwide frequency of sixteen major cancers in 1980. Int J Cancer 41: 184-197, 1988.

2. Parker SL, Tong T, Bolden S and Wingo PA: Cancer statistics. CA Cancer J Clin 46: 5-27, 1996.

3. Carlin BI and Andriole GL: The natural history, skeletal complications, and management of bone metastases in patients with prostate carcinoma. Cancer 88: 2989-2994, 2000.

4. Pentyala SN, Lee J, Hsieh K, Waltzer WC, Trocchia A, Musacchia L, et al: Prostate cancer: a comprehensive review. Med Oncol 17: 85-105, 2000.

5. Townsend MF, Sanders WH, Northway RO and Graham SD Jr: Bone fractures associated with luteinizing hormone-releasing hormone agonists used in the treatment of prostate carcinoma. Cancer 79: 545-550, 1997.

6. Collinson MP, Tyrell CJ and Hutton C: Osteoporosis occurring in two patients receiving LHRH analogs for carcinoma of the prostate (letter). Calcif Tissue Int 54: 327-328, 1994.

7. Daniell HW: Osteoporosis after orchiectomy for prostate cancer. J Urol 157: 439-444, 1997.

8. Clarke NW, McClure J and George NJ: The effects of orchiectomy on skeletal metabolism in metastatic prostate cancer. Scand J Urol Nephrol 27: 475-483, 1993.

9. Scher HI and Chung LW: Bone metastases: improving the therapeutic index. Semin Oncol 21: 630-656, 1994.

10. Berruti A, Dogliotti L, Bitossi R, Fasolis G, Gorzegno G, Bellina M, et al: Incidence of skeletal complications in patients with bone metastatic prostate cancer and hormone refractory disease: predictive role of bone resorption and formation markers evaluated at baseline. J Urol 164: 1248-1253, 2000.

11. Major P, Lortholary A, Hon J, et al: Zoledronic acid is superior to pamidronate in the treatment of hypercalcemia of malignancy: a pooled analysis of two randomized, controlled clinical trials. J Clin Oncol 19: 558-567, 2001.

12. Rosen LS, Gordon D, Tchekmedyian S, et al: Zoledronic acid versus placebo in the treatment of skeletal metastases in patients with lung cancer and other solid tumors: A phase III, doubleblind, randomized trial - The Zoledronic Acid Lung Cancer and Other Solid Tumors Study Group. J Clin Oncol 21: 3150-3157, 2003. 
13. Saad F, Gleason DM, Murray R, et al: A randomized, placebo-controlled trial of zoledronic acid in patients with hormone-refractory metastatic prostate carcinoma: for the Zoledronic Acid Prostate Cancer Study Group. J Natl Cancer Inst 94: 1458-1468, 2002.

14. Green JR, Müller K and Jaeggi KA: Preclinical pharmacology of CGP 42'446, a new, potent, heterocyclic bisphosphonate compound. J Bone Miner Res 9: 745-751, 1994.

15. Aapro M, Abrahamsson PA, Body JJ, et al: Guidance on the use of bisphosphonates in solid tumours: recommendations of an international expert panel. Ann Oncol 19: 420-432, 2008.

16. Vogel CL, Yanagihara RH, Wood AJ, et al: Safety and pain palliation of zoledronic acid in patients with breast cancer prostate cancer, or multiple myeloma who previously received bisphosphonate therapy. Oncologist 9: 687-695, 2004.

17. Rosen LS, Gordon D, Antonio BS, et al: Zoledronic acid versus pamidronate in the treatment of skeletal metastases in patients with breast cancer or osteolytic lesions of multiple myeloma: a phase III, double-blind, comparative trial. Cancer J 7: 377-387, 2001.
18. Saad F, Gleason DM, Murray R, et al: Long-term efficacy of zoledronic acid for the prevention of skeletal complications in patients with metastatic hormone-refractory prostate cancer. J Natl Cancer Inst 96: 879-882, 2004.

19. Saad F: Clinical benefit of zoledronic acid for the prevention of skeletal complications in advanced prostate cancer. Clin Prostate Cancer 4: 31-37, 2005.

20. Saad F: Bisphosphonates can prevent skeletal complications of malignant bone disease from prostate cancer and renal cell carcinoma. Eur Urol Suppl 6: 683-688, 2007.

21. Gulley JL, Wu S, Arlen PM and Dahut WL: Persistent hypocalcemia induced by zoledronic acid in a patient with androgen-independent prostate cancer and extensive bone metastases. Clin Genitourin Cancer 5: 403-405, 2007.

22. Bagan JV, Murillo J, Jimenez Y, et al: Vascular jaw osteonecrosis in association with cancer chemotherapy: series of 10 cases. J Oral Pathol Med 34: 120-123, 2005.

23. Vordos D, Paule B, Vacherot F, et al: Docetaxel and zoledronic acid in patients with metastatic hormone-refractory prostate cancer. BJU Int 94: 524-527, 2004. 